NASA-CR-200297

-oly'echinic

Modeling and Numerical Simulation of Microwave Pulse Propagation in Air Breakdown Environment

Technical Report for the work supported by AFGL through

NASA Grant No. NAG5-1051

S.P. Kun

Principal Investigator

J. Kim 


\section{1-1. Introduction}

There has been considerable interest in the generation of an artificial ionospheric mirror (AIM) by high power microwaves ${ }^{1-3}$. Using an AIM in the upper atmospheric region as a reflector of radio signals for over-thehorizon $(\mathrm{OTH})$ communications ${ }^{4}$ or radar purposes ${ }^{5}$ has several advantages over the conventional approach of using the ionosphere as a reflector. 1 . The AIM can be produced at a much lower altitude and yet cover a large range by titling the mirror, 2 . it is able to reflect radio signals of much higher frequency through the ionosphere and, 3. its stability and location are controllable. Moreover, as shown in Fig.1-1 AIM can be used to cover the skip zone, which is required to avoid clutter in the radar return of the OTH radar.

The study of the AIM involves both intense ground-transmitted microwave pulse propagation and air-breakdown phenomenon for creating localized patches of ionization (AIM) in the stratospheric/ mesospheric altitude range. When air-breakdown is produced by an intense microwave, a variety of physical processes can result. Among these the most significant is the ionization process. This occurs when the existing free electrons gain an kinetic energy of greater than $12 \mathrm{eV}$. Moreover, when free electrons are created more rapidly than their loss due to attachment, recombination and diffusion, their density quickly builds up through the process of cascading 


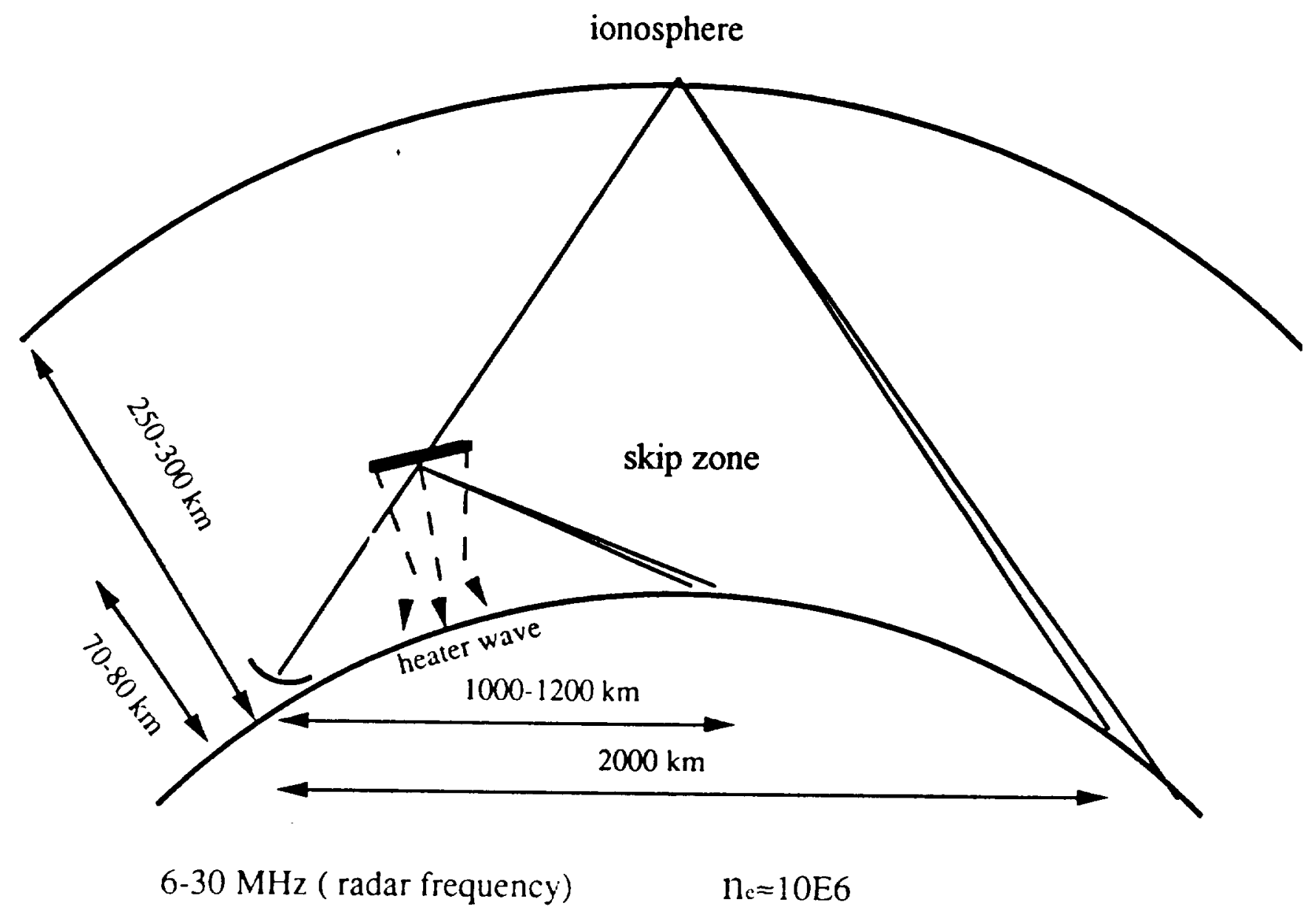

Fig.1-1 OTH radar using artificial plasma patch as a mirror. 
breakdown in the background gas. These electrons then absorb and/or reflect the energy of the microwave pulses, leading to serious attenuation of pulse energy (so called "tail erosion") ${ }^{6-11}$. Consequently, the eroded pulse may not be able to produce desirable ionization in the altitude region interesting to AIM applications. In the present work the propagation of intense microwave pulses through the atmosphere is studied numerically via a theoretical model which has been validated by the chamber experiment.

The study is concerned with two interrelated fundamental issues; one involves the propagation characteristics of the microwave pulse in the selfinduced space-time dependent plasma. The second is related to the determination of the optimum pulse parameters for maximum energy transfer through the background gas. Therefore, a wide range of pulse parameters including intensity, frequency, width, and shape, etc. are considered in the numerical simulation study in relation to the above issues.

The theoretical model of pulse propagation used in the present work is an extension of the previous one ${ }^{12}$ which was developed for describing the chamber experiment ${ }^{11}$. This extension is necessary because using the previous model to the problem of intense microwave pulse propagation through atmosphere will require a tremendous computer memory and running time. The new model employs a transformation to the local time frame of reference to convert the original modal equations of partial differential equation (PDE) form into a new set of modal equations of ordinary differential equation (ODE) form. The new set of equations can be solved directly by the available subroutine of an ODE solver ${ }^{13}$, reducing the computation time considerably.

The previous model is also incomplete as the ionization loss is neglected and the possible focusing effect is not included. Though the 
shortcoming of that model did not affect the outcome of the comparison with the chamber experiments, their effect on pulse propagation in the atmosphere can be important. Thus, in the present work the previous model is further improved by properly including the ionization loss and the focusing effect. The new code is then employed to perform a parametrized study of pulse propagation in the atmosphere.

Presented in section 1-2 is the theoretical model and the set of governing equations for the intense microwave pulse propagation in the air. The results of numerical simulations are presented in section 1-3. A summary of the work and potential applications are given in section 1-4. 


\section{1-2. Theoretical Model}

The propagation of an intense electromagnetic pulse through atmosphere is considered. The plasma along the path of the pulse is selfgenerated and causes the erosion of the pulse. The microwave-generated plasma is described by the electron density rate equation

$$
\frac{\partial}{\partial} \mathrm{n}=\left(v_{\mathrm{i}}-v_{\mathrm{a}}\right) \mathrm{n}-\mathrm{m}^{2}
$$

and the electron momentum equation

$$
\mathrm{m}_{\mathrm{e}} \frac{\partial}{\partial} \mathrm{n} \overrightarrow{\mathrm{v}}=-\mathrm{ne} \overrightarrow{\mathrm{E}}-\left(\eta v_{\mathrm{i}}+v_{\mathrm{a}}+\eta \mathrm{n}+v\right) \mathrm{m}_{\mathrm{e}} \mathrm{n} \overrightarrow{\mathrm{v}}
$$

where $v_{\mathrm{i}}, v_{\mathrm{a}}, \gamma$, and $v$ are the ionization frequency, attachment frequency, recombination coefficient, and electron-neutral particle collision frequency respectively. The ionization energy is represented by $\varepsilon_{i}$, and $\eta=\left(2 \varepsilon_{i} / 3 T_{e}\right)^{1 / 2}$ is a measure of the effective momentum loss of the plasma fluid in each ionization process. Since the time scale considered is much shorter than the diffusion time, the diffusion term in (1-1) and the convective term in (1-2) are neglected.

With the aid of (1-1), Eq. (1-2) reduces to

$$
\frac{\partial}{\partial} \overrightarrow{\mathbf{v}}=-\mathrm{e} \overrightarrow{\mathrm{E}} / \mathrm{m}_{\mathrm{e}}-\left[(\eta+1) v_{\mathrm{i}}+v\right] \overrightarrow{\mathrm{v}}
$$


The wave equation derived from the Maxwell's equations is given by

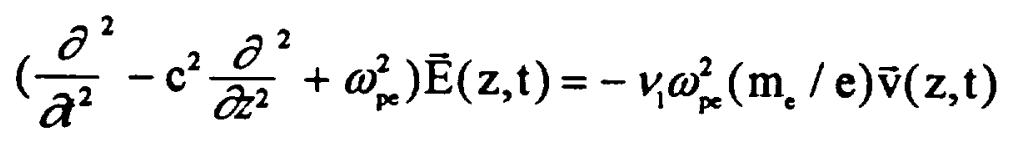

where the relation $\overrightarrow{\mathrm{J}}=-\mathrm{en} \overrightarrow{\mathrm{v}}$ has been used, $v_{1}=\eta v_{\mathrm{i}}+v_{\mathrm{a}}+\gamma \mathrm{n}+v_{\text {, and }}$ $\omega_{p e}=\left(4 \pi e^{2} / m_{c}\right)^{1 / 2}$ is the electron plasma frequency.

Equations (1-1), (1-3), and (1-4) provide a self-consistent description of pulse propagation in an ionizing background. They are coupled through the ionization frequency $v_{i}$, which is modeled to be $\mathrm{e}^{14}$

$$
v_{i}=3.83 \times 10^{2} v_{i}\left(\varepsilon^{3 / 2}+3.94 \varepsilon^{1 / 2}\right) \exp (-7.546 / \varepsilon)
$$

where $\varepsilon=\left|\mathrm{A} / \mathrm{A}_{\mathrm{th}}\right|$ is the wave field normalized to the breakdown (ionization) threshold field, $A_{t h}$. The expression $E(z, t)=A(z, t) e^{-i \neq(z, t)}+c . c$. for wave field is assumed, where c.c. representing the complex conjugate. For a $\mathrm{cw}$ wave, $\mathrm{A}_{\mathrm{th}} \approx 18 p\left(1+\omega^{2} / v^{2}\right)^{1 / 2} \mathrm{~V} / \mathrm{cm}$ where $\mathrm{p}$ is the background pressure measured in Torr and $\omega$ is the angular frequency of the wave.

If the pulse is not too short, i.e. it contains many oscillations, slow time and spatial varying envelope approximations can be used to analyze (1-3) and (1-4). This is done by first setting $v(z, t)=V(z, t) e^{-i \phi(z, t)}+c . c$. and $E(z, t)=\left[A^{\prime}(z, t) /(1-z / L)\right] e^{-i \alpha z, t)}+$ c.c., where $A^{\prime}$ and $\phi$, are real functions and $A^{\prime} /(1-z / L)=A, L$ is the focal length of the pulse, $L \rightarrow \infty$ for an unfocused pulse beam. $V(z, t)$ is, in general, a complex function and the variation of the amplitude function $A^{\prime}(z, t)$ and $|V(z, t)|$ in space and time is much slower than that of the phase function $\phi(z, t)$. These expressions for $v(z, t)$ and $E(z, t)$ are then substituted into (1-3) and (1-4). Using the relations 
$\left|\partial \ln A^{\prime} / \partial\right| \ll|\partial \phi / \partial|,\left|\partial \ln A^{\prime} / \partial z\right| \ll|\partial \phi / \partial z|$, and $|\partial \ln V / \partial|<|\partial \phi / \partial|$, as well as employing the forward-scattering approximation giving the definitions of local frequency $\omega=\partial \phi / \partial$, and local wave number $k=-\partial \phi / \partial z,(1-3)$ and (1-4) are simplified considerably. Next, Eq (1-3) is solved to yield $\mathrm{V}(\mathrm{z}, \mathrm{t}) \approx-\mathrm{ieA}(\mathrm{z}, \mathrm{t}) / \mathrm{m}_{\mathrm{e}}\left(\omega+\mathrm{i} v_{2}\right)$, where $v_{2}=(\eta+1) v_{\mathrm{i}}+v$. This result is used to reduce (1-4) into a Poynting equation

$$
\frac{\partial}{\partial} \mathrm{P}+\frac{\partial}{\partial \mathrm{z}} \mathrm{v}_{\mathrm{B}} \mathrm{P}=-\beta \mathrm{P}+2 \mathrm{v}_{\mathrm{g}} \mathrm{P} /(\mathrm{L}-\mathrm{z})
$$

where $P=A^{2} / 2 \pi$ is the energy density of the pulse, $v_{g}=\partial \omega / \partial \mathrm{k}$ is the group velocity, and $\beta=\nu_{1} \omega_{\mathrm{pe}}{ }^{2} /\left(\omega^{2}+\nu_{2}^{2}\right)$.

The focusing model is based on the continuity of the power flux. Diffraction is neglected in modeling the focusing effect of the microwave beam. This model can be easily generalized to include a finite focusing spot size by replacing $(1-z / L)$ by $\left[(1-z / L)^{2}+\Delta^{2}\right]^{1 / 2}$, where $\Delta$ is the ratio of the cross section area of the beam at the focal height $z=L$ to the emitting area of the antenna. The simpler model is chosen as the two models give negligibly different results in the region below the focal layer.

The other equation deduced from $(1-4)$ is the real part of the local dispersion relation of the pulse, given by

$$
\omega^{2}=\mathrm{k}^{2} \mathrm{c}^{2}+\omega_{p e}^{2}\left[1-v_{1} v_{2} /\left(\omega^{2}+v_{2}^{2}\right)\right]
$$

which is used to determine the local group velocity $v_{z}=\partial \omega / 2 \mathrm{k}$ of the pulse. 
In the following analysis, (1-1) and (1-6) are the set of modal equations giving a self-consistent description of pulse propagation in a self-generated plasma. Their coupling is through the ionization frequency given by equation (1-5), which is reexpressed in terms of $\bar{P}=P / P_{a}$ as

$$
v_{\mathrm{i}} / p=2.5 \times 10^{7}\left[8.8 \overline{\mathrm{P}}^{1 / 4}+2.236 \overline{\mathrm{P}}^{3 / 4}\right] \exp \left[-7.546 / \overline{\mathrm{P}}^{1 / 2}\right](\mathrm{sec}-\mathrm{Tor})^{-1}
$$

where $\mathrm{P}_{\mathrm{c}}=\mathrm{E}_{\mathrm{a}}{ }^{2} / 8 \pi=\mathrm{A}_{\mathrm{th}}{ }^{2} / 2 \pi$ and $\mathrm{E}_{\mathrm{c}}=2 \mathrm{~A}_{\mathrm{th}}=36.84 p\left(1+\omega^{2} / v^{2}\right)^{1 / 2} \mathrm{~V} / \mathrm{cm}$ is the breakdown threshold field of the background air of pressure $p$.

When considering the practical application of pulse propagation over a long distance, the required computation time becomes an important issue. The computation time in solving the pair of coupled equations (1-1) and (1-6) is long because the form of (1-6) which contains both spatial and temporal derivative terms. Hence, a transformation to the local time frame of reference is introduced to separate the spatial and temporal derivative from the same equation $^{15}$. In doing so, the computation time for the same propagation distance can be reduced tremendously.

The frame transformation is defined to $\mathrm{be}^{12}$

$$
t=t^{\prime}+\int_{0}^{z} d z^{\prime \prime} / V_{B}\left(t^{\prime}, z^{\prime \prime}\right)
$$

and

$$
\mathbf{z}=\mathbf{z}^{\prime}
$$

where $t^{\prime}$ is the local time of the pulse and $V_{g}\left(t^{\prime}, z^{\prime}\right)=v_{g}(t, z)$. 
Thus (1-6) and (1-1) become

$$
\frac{\partial}{\partial z^{\prime}} V_{s} P_{1}\left(1-z^{\prime} / L\right)^{2}=-\left[v_{1}+c^{2} v_{1} B / 2 V_{z}^{2}\right]\left(1-z^{\prime} / L\right)^{2} P_{1} N / n_{c}
$$

and

$\frac{\partial}{\partial t^{\prime}} N=v_{.} B N\left(V_{80} P_{10} / V_{8} P_{1}\right)\left(1-z^{\prime} / L\right)^{-2} \exp \left[-v_{1} \int_{0}^{z^{\prime}} d z^{\prime \prime}\left(N / n_{c}\right) / V_{8}\right]$

where $P_{1}\left(t^{\prime}, z^{\prime}\right)=P(t, z), N\left(t^{\prime}, z^{\prime}\right)=n(t, z), \quad V_{80}=V_{g}\left(t^{\prime}, z^{\prime}=0\right), \quad P_{10}=P_{1}\left(t^{\prime}\right.$, $\left.\mathrm{z}^{\prime}=0\right), \overline{\mathrm{P}}_{1}=\mathrm{P}_{1} / \mathrm{P}_{\mathrm{a}}$, and

$$
\mathrm{B}=v_{\mathrm{i}} / v_{\mathrm{a}}-1=3.83 \times 10^{2}\left(\overline{\mathrm{P}}_{1}^{3 / 4}+3.94 \overline{\mathrm{P}}_{1}^{1 / 4}\right) \exp \left(-7.546 / \overline{\mathrm{P}}_{1}^{1 / 2}\right)-1
$$

In the above equations, $v, v_{i}, v_{a}, P_{c r}$, and $n_{c}$ are all altitude dependent, and their functional forms are determined as follows. Assuming that the air pressure at $50 \mathrm{Km}$ height is 1 Torr, and the pressure decays exponentially with the altitude $z^{\prime}$, the pressure is given by $p\left(z^{\prime}\right)=760 \mathrm{e}^{-1.346 \times 10^{-4} z^{\prime}}$ Torr, where $\mathrm{z}^{\prime}$ is measured in meters, and $v=v_{0} \mathrm{e}^{-1.346 \times 10^{-4} \mathrm{z}^{\prime}}$, where $v_{0}=v\left(\mathrm{z}^{\prime}=0\right)$. In terms of the ground level dc breakdown threshold power $P_{c 0}=P_{\sigma}\left(z^{\prime}=0, \omega=0\right)$, then $\mathrm{P}_{\mathrm{c}}\left(\mathrm{z}^{\prime}\right)=\mathrm{P}_{\mathrm{c} 0}\left(\eta+\mathrm{e}^{-2.692 \times 10^{-1} \mathrm{z}^{\prime}}\right)$, where $\eta=\omega^{2} / v_{0}^{2}$. Similarly, $\mathrm{n}_{\mathrm{c}}\left(\mathrm{z}^{\prime}\right)=\mathrm{n}_{\mathrm{c} 0}(\eta+$ $\left.\mathrm{e}^{-2.692 \times 10^{-4} z^{\prime}}\right)$, where $\mathrm{n}_{\mathrm{co}}=\mathrm{m}_{\mathrm{e}} v_{0}^{2} / 4 \pi \mathrm{e}^{2}$. At 1 Torr pressure, $v_{\mathrm{a}}=v_{\mathrm{cc}}=$ $1.457 \times 10^{5} \mathrm{sec}^{-1}$ and $v=v_{\mathrm{s}}=6 \times 10^{9} \mathrm{sec}^{-1}$ for $1 \mathrm{eV}$ electron temperature ${ }^{2}$. Including the effect of electron heating by the microwave pulse and assuming that the unperturbed background temperature is about $0.03 \mathrm{eV}$, a modal function for $v$ is given by $v=v_{c} p\left[\overline{\mathrm{P}}_{1}^{1 / 2}+0.03\right]^{1 / 2}$, where $\overline{\mathrm{P}}_{1}=\mathrm{P}_{1} / \mathrm{P}_{\mathrm{cr}}=$ 
$\left(P_{1} / P_{\infty}\right)\left(\eta+e^{-2.692 \times 10^{-4} z^{\prime}}\right)^{-1}$. We now introduce the dimensionless variables and functions: $\quad\left(\nu_{\mathrm{c}} \nu_{\mathrm{sc}}\right)^{1 / 2} \mathrm{t}^{\prime} \rightarrow \mathrm{t}, \quad\left(\nu_{\mathrm{c}} \nu_{\mathrm{sc}}\right)^{1 / 2} \mathrm{z}^{\prime} / \mathrm{c} \rightarrow \mathrm{z}, \quad\left(\nu_{\mathrm{c}} \nu_{\mathrm{\alpha c}}\right)^{1 / 2} \mathrm{~L} / \mathrm{c} \rightarrow \mathrm{L}$, $\overline{\mathrm{n}}=\mathrm{N} / \mathrm{n}_{\mathrm{c}}=\left(\mathrm{N} / \mathrm{n}_{\mathrm{co}}\right) \mathrm{h}(\mathrm{z}) \rightarrow \mathrm{n}$, where $\mathrm{h}(\mathrm{z})=\left(\eta+\mathrm{e}^{-2.692 \times 10^{-4} \mathrm{z}}\right)^{-1}, \quad \overline{\mathrm{P}}_{1} \rightarrow \mathrm{P}_{1}$, $\overline{\mathrm{P}}_{10}=\mathrm{P}_{1} / \mathrm{P}_{c 0} \rightarrow \mathrm{P}_{10}, \quad \mathrm{Q}=(1-\overline{\mathrm{n}})^{1 / 2} \overline{\mathrm{P}}_{10}\left(1-\mathrm{z}^{\prime} / \mathrm{L}\right)^{2} \rightarrow(1-\mathrm{n})^{1 / 2} \mathrm{P}_{10}(1-\mathrm{z} / \mathrm{L})^{2}$, $P_{10}=Q /(1-n)^{1 / 2}(1-z / L)^{2}$, and $P_{1}=P_{10} h(z)=\left[Q /(1-n)^{1 / 2}(1-z / L)^{2}\right] h(z)$. In terms of these dimensionless variables and functions, we have the numerical values $\left(v_{c} / v_{x c}\right)^{1 / 2}=203$ and $\left(v_{c} v_{x}\right)^{1 / 2}=2.957 \times 10^{7} \sec ^{-1}$, and Eqs. $(1-9)-(1-11)$ become

$$
\begin{gathered}
\frac{\partial}{\partial t} \mathrm{n}=3.745 \mathrm{~g}(\mathrm{z}) \mathrm{Bn}\left(\mathrm{Q}_{0} / \mathrm{Q}\right) \exp \left[-1.54 \times 10^{5} \int_{0}^{\mathrm{z}} \mathrm{f}(\mathrm{x}) \mathrm{dx}\right] \\
\frac{\partial}{\partial \mathrm{z}} \mathrm{Q}=-3.745 \mathrm{~g}(\mathrm{z})\left\{(203)^{2}\left[\left(\mathrm{P}_{10} \mathrm{~h}\right)^{1 / 2}+0.03\right]^{1 / 2}+\mathrm{B} / 2(1-\mathrm{n})\right\} \mathrm{nQ} / \sqrt{1-\mathrm{n}}
\end{gathered}
$$

and

$$
\mathrm{B}=3.83 \times 10^{2}\left\{\left(\mathrm{P}_{10} \mathrm{~h}\right)^{3 / 4}+3.94\left(\mathrm{P}_{10} \mathrm{~h}\right)^{1 / 4}\right\} \exp \left[-7.546 /\left(\mathrm{P}_{10} \mathrm{~h}\right)^{1 / 2}\right]-1
$$

where $g(z)=e^{-1.346 \times 10^{-4} z}, f(x)=g(x)\left\{\left[P_{10}(x) h(x)\right]^{1 / 2}+0.03\right\}^{1 / 2}[n(x) / \sqrt{1-n(x)}$ ], and $\mathrm{Q}_{0}=\mathrm{Q}(\mathrm{t}, \mathrm{z}=0)$.

Equations (1-12) and (1-13) describe vertical propagation of microwave pulse through the atmosphere either from ground to space or from space to ground. If the pulse propagates obliquely with an angle $\theta$ with respect to the vertical axis, (1-12) and (1-13) must be corrected by replacing $\mathrm{V}_{\mathrm{g}}$ with $\mathrm{V}_{\mathrm{g}} \cos \theta$, i.e., replacing $\sqrt{1-\mathrm{n}}$ by $\sqrt{1-\mathrm{n}} \cos \theta$ in these two equations. 
In terms of $z$ and $t$, the real distance $x$ and time $\tau$ are given by $x=10.1 z(m)$ and $\tau=33.82 \mathrm{t}$ (nsec). 


\section{1-3. Numerical Simulation Results}

The dependencies of the propagation characteristics of an unfocused rectangular pulse (i.e. $L \rightarrow \infty$ ) on its power, width and carrier frequency are first investigated by integrating the modal equations numerically. The pulse is assumed to propagate vertically upwards from the ground. When the pulse reaches the region where the breakdown threshold is exceeded by the pulse power, plasma is generated and causes erosion of the tail part of the pulse. This tail erosion phenomenon at four different altitude locations (46.04, $50.75,52.78$ and $56.84 \mathrm{~km}$ ) is demonstrated in Fig.1-2(a). At the height of $46.04 \mathrm{~km}$ the breakdown threshold is only slightly exceeded, producing very little plasma in the region below this height. Consequently the pulse is almost unperturbed. At the three higher locations, the pulse has interacted with increasingly more plasma, and its tail erosion increases accordingly as shown.

The corresponding electron densities at the four locations are presented in Fig.1-2(b). The graph shows the electron density at $46.04 \mathrm{~km}$ grows slowly to a very low saturation value. However, at a higher altitude, e.g. $50.75 \mathrm{~km}$, the breakdown threshold becomes lower. A stronger breakdown leads to a faster build-up of electron density to a much higher saturation level of about $8 \times 10^{5} \mathrm{~cm}^{-3}$. The saturation of the electron density is due to the tail erosion effect. A further increase of the altitudes, e.g., at 52.78 and 56.84 $\mathrm{km}$, the breakdown threshold is reduced, but the powers (widths) of the pulse at these two locations drops even faster due to the tail erosion process. Therefore, the electron densities at those two locations grow more quickly, but saturate at lower levels.

The distribution of the maximum (in time) electron density along the trail of the pulse is presented in Fig.1-2(c). This graph shows that significant 


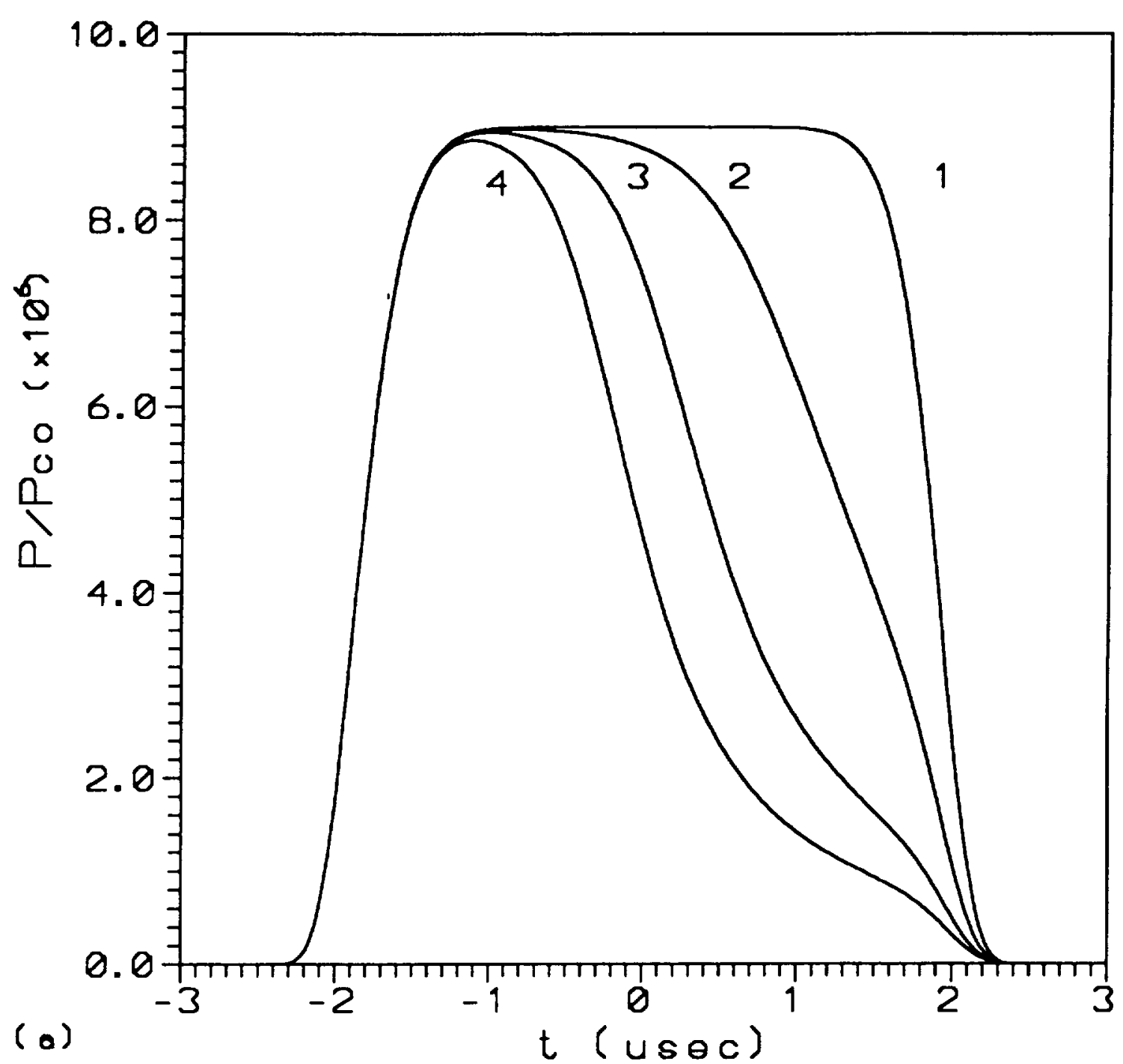

Fig.1-2 Propagation of an unfocused 5.4 $\mu$ sec pulse having $1 \mathrm{GHz}$ carrier frequency and an initial power intensity $\mathrm{P}=9 \times 10^{-6} \mathrm{P}_{\mathrm{co}}$

(a) Tail erosion of pulse propagating to four altitudes: 1. 46.24, 2. $50.75,3.52 .78$, and $4.56 .84 \mathrm{~km}$. 


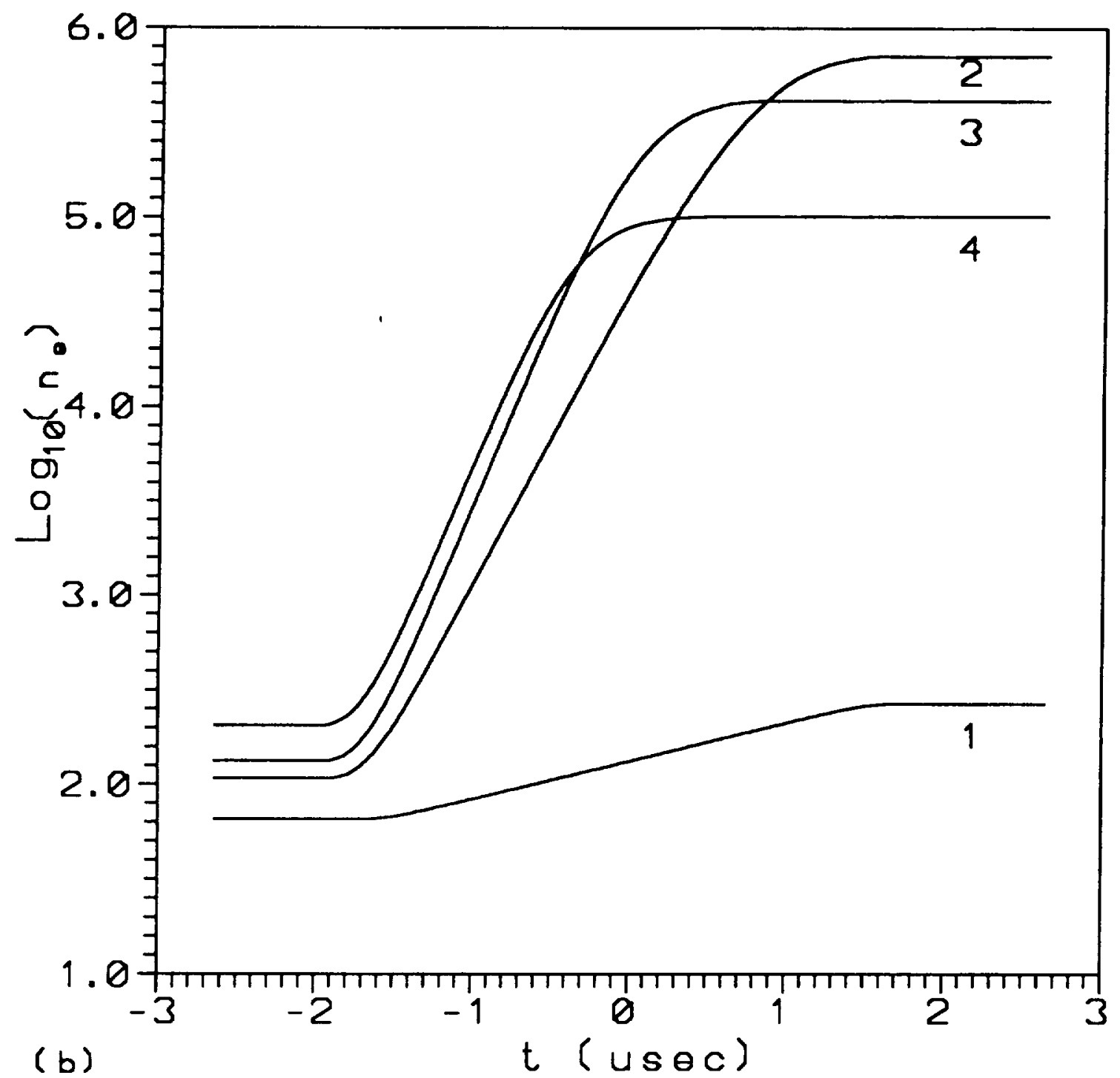

Fig.1-2 (b) Electron density $\left(\mathrm{cm}^{-3}\right)$ vs time at the four locations considered in (a). 


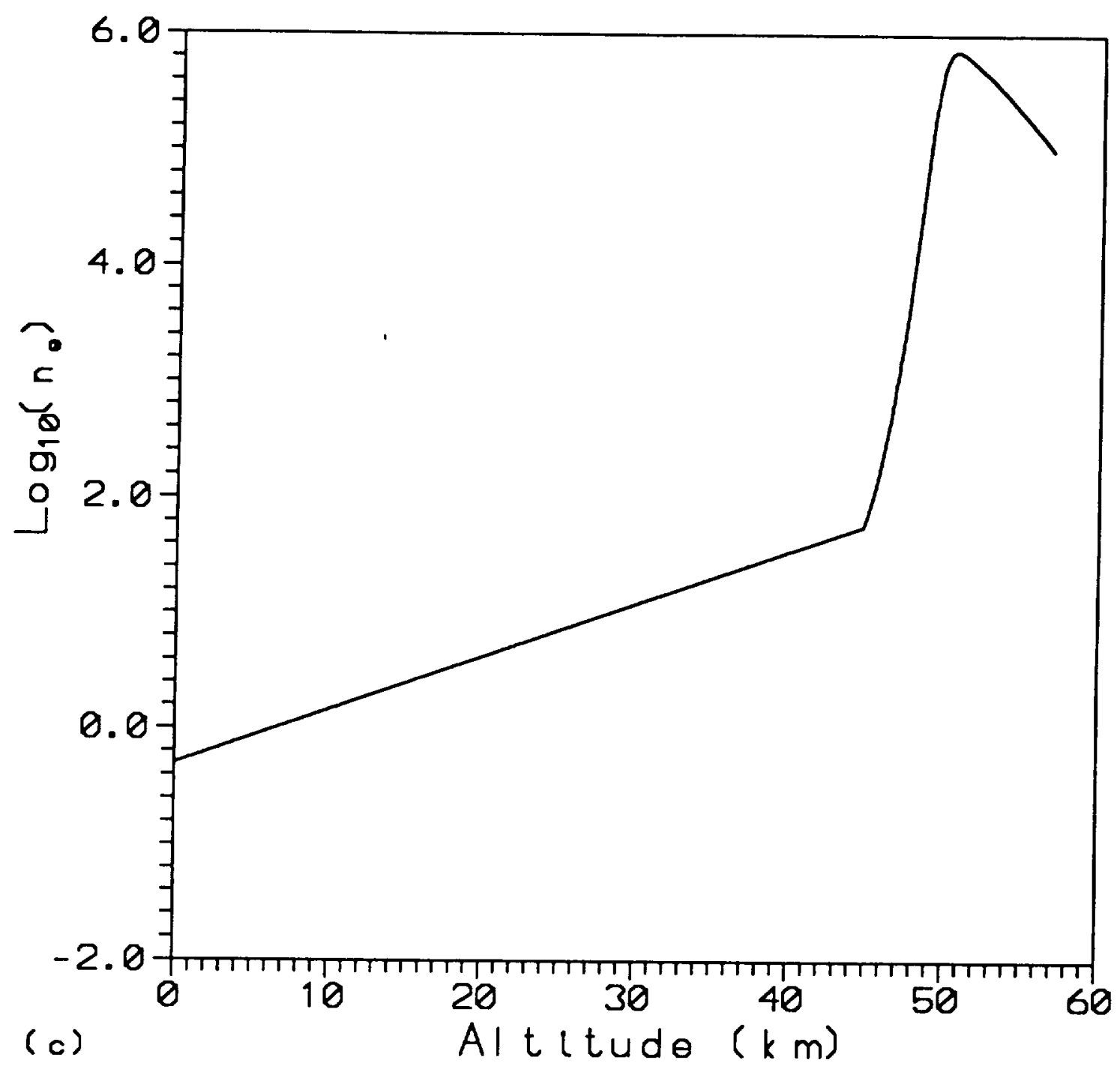

Fig.1-2 (c) Distribution of the maximum (in time) electron density $\left(\mathrm{cm}^{-3}\right)$ along the path of the pulse. 
ionization occurs only in a very small spatial region attributed to tail erosion effect. These results suggest that the propagation characteristics of a high power microwave pulse be closely related to the electron density along its propagation path. Presented in Fig.1-3(a) are the electron density distributions along the path of the pulses for four different incident power levels: $7 \times 10^{-6}, 9 \times 10^{-6}, 5 \times 10^{-5}$ and $10^{-4}$ of the ground level breakdown threshold power. As shown, an increase of pulse power enhances the level of the peak electron density but also lowers the region of cascading breakdown. In other words, the pulse is eroded earlier and more severely as its power level is increased. The power levels of the four pulses in Fig.1-3(a) at their maximum electron density locations are evaluated respectively to be 2.608 , $2.504,2.286$ and 1.553 of the corresponding local breakdown threshold power.

One possible way to reduce tail erosion of a high power pulse is to increase the carrier frequency and width of the pulse together. The classical breakdown threshold curves for optimized pulse widths indicate that the breakdown threshold field increases with the carrier frequency $\omega$ in the dependence of $\left(1+\omega^{2} / v^{2}\right)^{1 / 2}$ and has a minimum at $\omega=v$. The appearance of this minimum is due to the transit time effect of short pulse width (i.e. Paschen effect). If the pulse is long enough, the breakdown threshold curves tend to become flat at higher altitude and the minimum disappears. Using a long pulse width of $5.4 \mu \mathrm{sec}$ and fixing the incident power level of $9 \times 10^{-6}$ of the breakdown threshold power, but increasing the carrier frequency of the pulse from $0.5 \mathrm{GHz}$ to $1.5 \mathrm{GHz}$, as demonstrated in Fig.1-3(b) the breakdown location of the pulse moves upwards (instead of downwards) and ionization decreases. However, this approach may not be feasible because the required frequency increase is proportional to the field intensity of the pulse. 


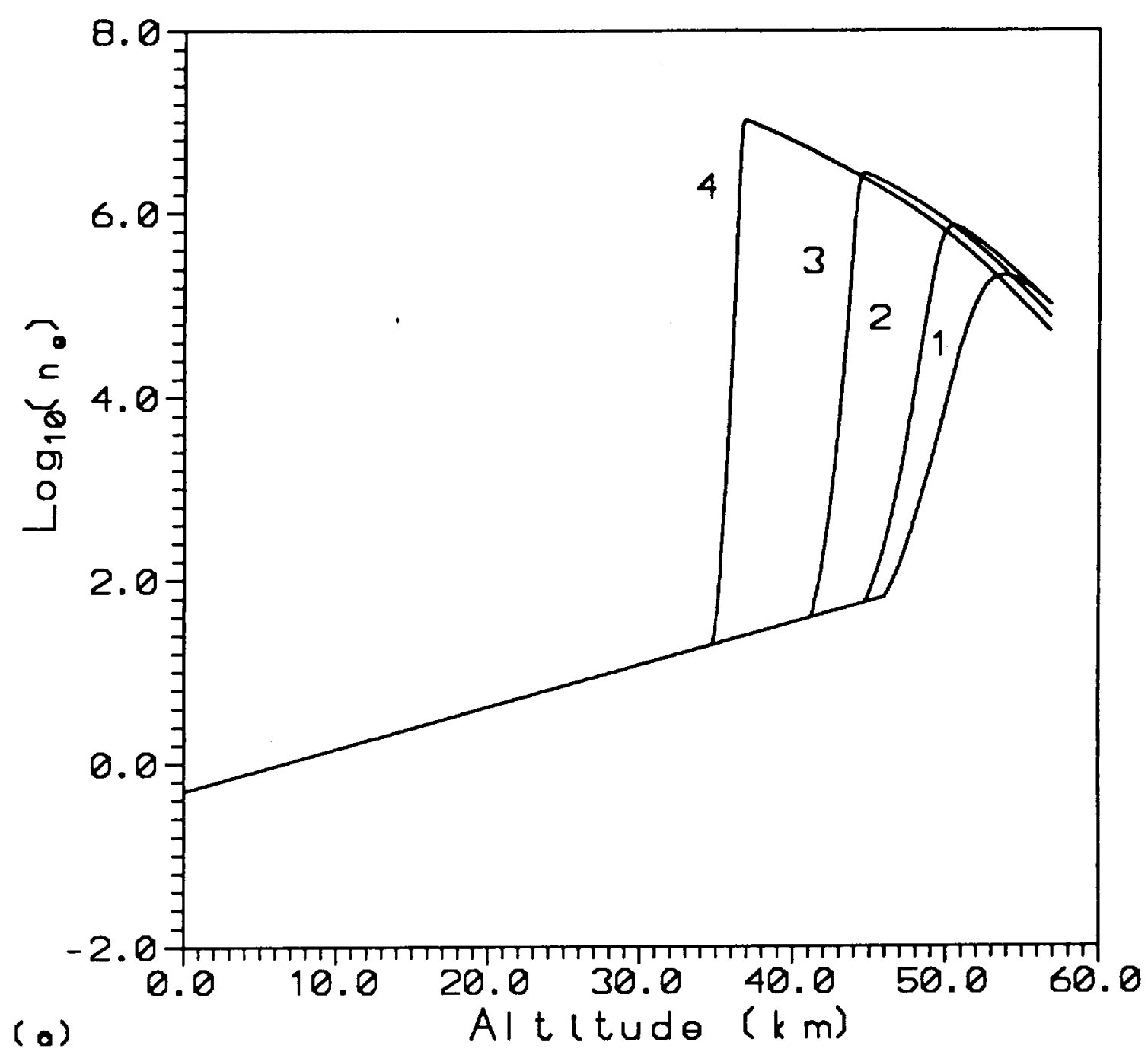

Fig.1-3 Spatial distribution of electron density $\left(\mathrm{cm}^{-3}\right)$

(a) The maximum (in time) electron density $\left(\mathrm{cm}^{-3}\right)$ distribution generated by a pulse $(5.4 \mu$ s and $1 \mathrm{GHz})$ for four incident powers $\left(\mathrm{P} / \mathrm{P}_{\mathrm{co}}\right): 1.7 \times 10^{-6}, 2.9 \times 10^{-6}, 3.5 \times 10^{-5}$, and $4.1 \times 10^{-4}$. 
3

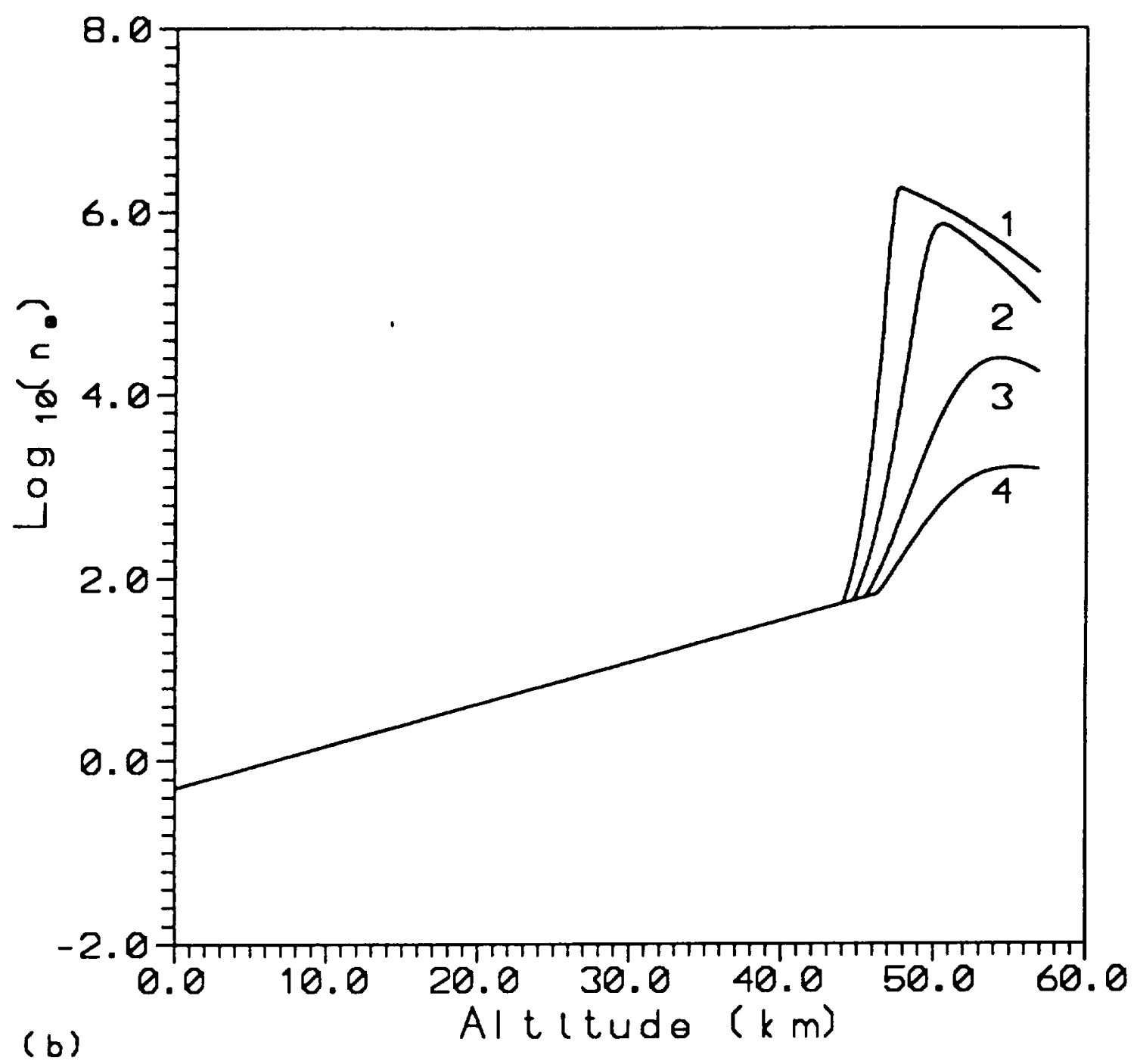

Fig.1-3 (b) The maximum (in time) electron density $\left(\mathrm{cm}^{-3}\right)$ distribution for four carrier frequencies: $1.0 .5,2.1,3.1 .3,4.1 .5 \mathrm{GHz}$. 
The dependence of the ionization characteristics on pulse width is also examined. The presented in Fig.1-3(c) demonstrate that the location of cascading breakdown is not sensitive to the pulse width. In other words, the Paschen effect disappears as the pulse width is wide enough. However, the longer the pulse is the higher the peak level of the electron density, and the thicker the ionization layer. The numerical results also indicate that the shape of the pulse is not an important factor in determining the electron density distribution and the location of cascading breakdown. This is because a change of the pulse shape has the same effect as changing the pulse width.

An empirical relationship between the pulse width and pulse power for a fixed percentage of energy transfer from ground to a given altitude is determined. As shown in Fig. 1-4, three constant percentages of pulse energy $(60 \%, 68 \%, 90 \%)$ transmitted to a desired altitude (e.g. $50 \mathrm{~km})$ depend all approximately on the product of the cubic power of the normalized initial pulse power $\left(\mathrm{P}^{3}\right)$ and the pulse width $(\mathrm{W})$, i.e. $\mathrm{P}^{3} \mathrm{~W}=$ constant which is proportional to the percentage of pulse energy transferred from the source to a destined location. This shows that the percentage of energy transferred is a more sensitive to pulse power than the pulse width. Thus, without further treatment of the pulse, such as introducing a focusing phase, the maximum electron density produced by a single pulse at the altitude of interest (e.g. 50 $\mathrm{km}$ ) is found to be limited by the tail erosion effect to about $10^{6} \mathrm{~cm}^{-3}$.

We examine three different approaches in the following to determine if the ionization at the destined location can be improved. One uses a repetitive pulse approach, the second a focused pulse beam approach, and the third two intersecting beams. Using periodic pulses with $5.4 \mu$ s pulse width and a $50 \%$ duty cycle, the induced electron density distribution is presented in Fig.1-5, where only the electron densities resulting from the first three pulses are 


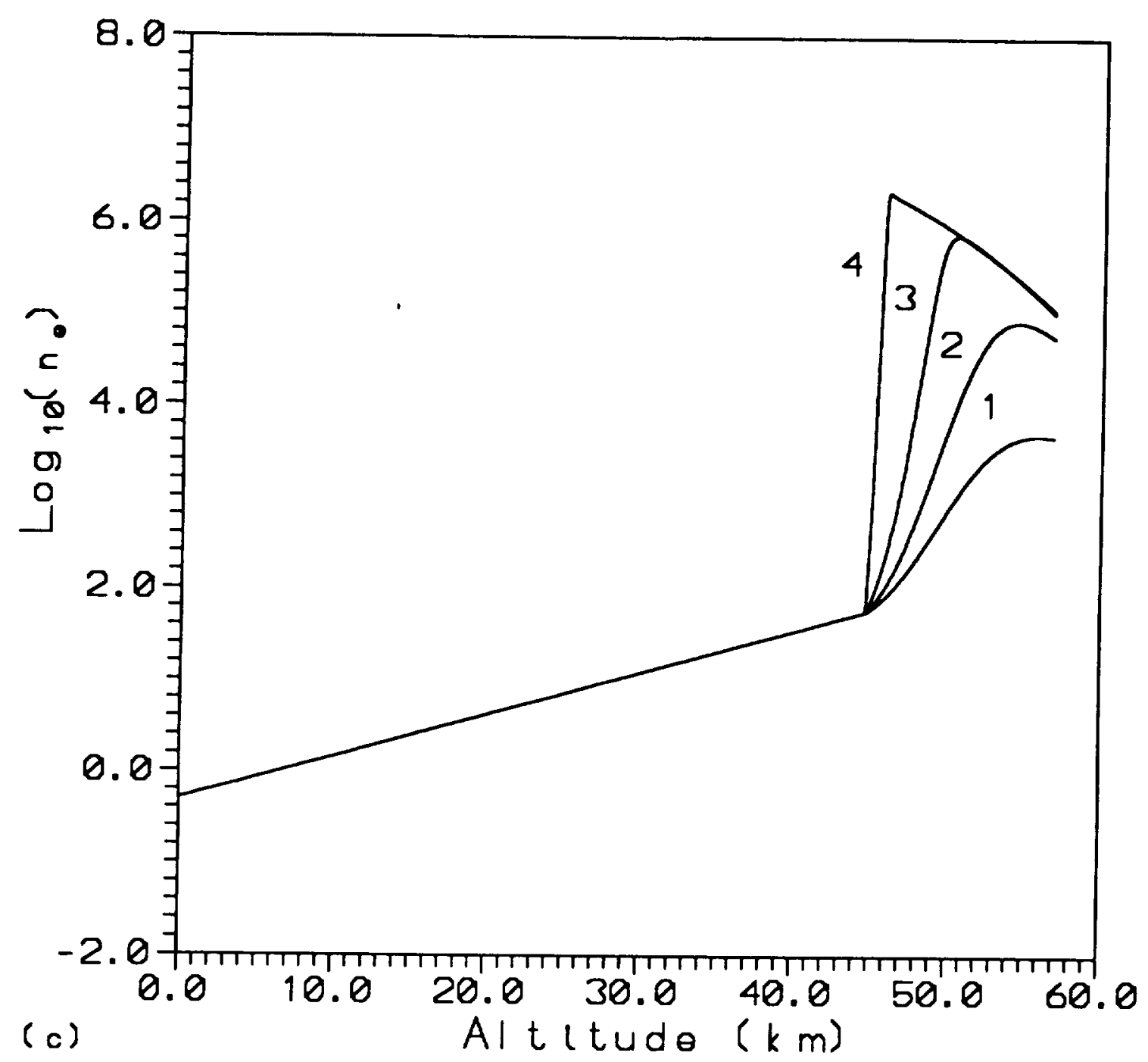

Fig.1-3 (c) The maximum (in time) electron density $\left(\mathrm{cm}^{-3}\right)$ distribution for four pulse widths: 1. 1.68, 2. 2.16, 3. 5.41 and 4. 54.1 $\mu \mathrm{s}$. 


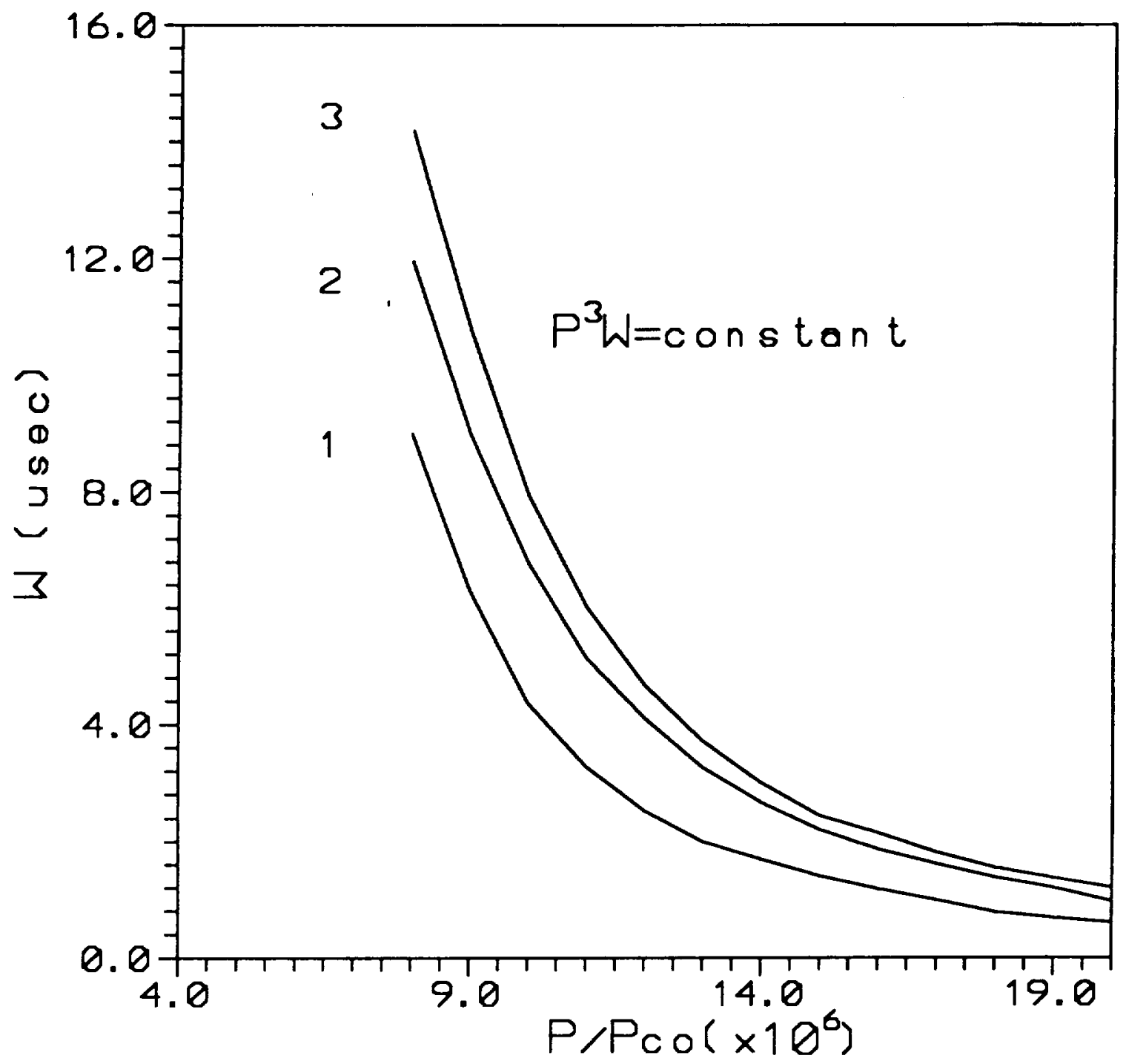

Fig.1-4 Relationship between pulse width $\mathrm{W}$ and power $\mathrm{P}$ leading to a constant percentage of pulse energy transferred from ground to $50 \mathrm{~km}$ altitude for three percentages: $1.90 \%, 2.68 \%, 3.60 \%$. The frequency of the pulse is $1 \mathrm{GHz}$. 


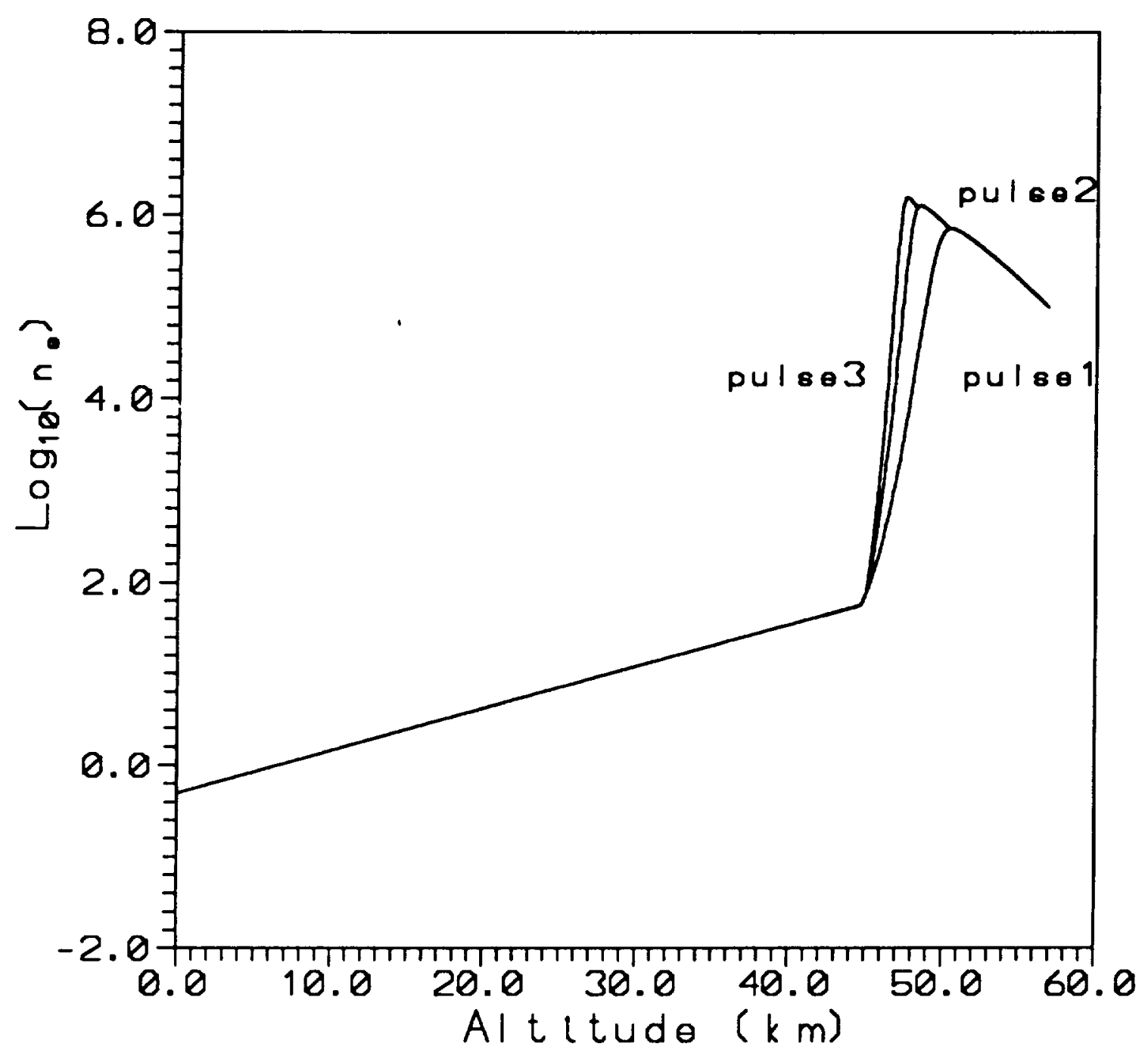

Fig.1-5 Electron density $\left(\mathrm{cm}^{-3}\right)$ distribution generated by repetitive pulses with a $50 \%$ duty cycle; up to three pulses are demonstrated. Frequency, width and normalized initial power of each pulse are $1 \mathrm{GHz}, 5.4 \mu \mathrm{s}$ and $9 \times 10^{-6}$, respectively. 
included, as the electron density distribution remains about the same after the third pulse. The results indicate that repetitive pulses can indeed increase electron density at the desired location, but it still saturates at a relatively low value due to the tail erosion effect.

If we assume for the second case that the focusing effect is limited to a power amplification factor of 10 at $50 \mathrm{~km}$ height (i.e. having $L \geq 73 \mathrm{~km}$ ) for practical reasons, the result presented in Fig.1-6 shows that the maximum electron density at the given location ( $50 \mathrm{~km}$ height) can only be increased by less than an order of magnitude (comparing with case 3 of Fig.1-6 and Fig.13(c)). The peak level is, again limited by the tail erosion effect and can not be enhanced significantly by the increase of the pulse width, as its main effect is to increase the spatial width of the ionization layer. Again, this density level is too low for the considered applications. It is noted that the electron density can be increased to the cutoff density level if the focusing effect is increased by another two orders of magnitude. However, this would be impractical as it requires a very large antenna array, and also the ionization region becomes too small to be a reflector.

We, therefore, explore a third alternative approach using two intersecting pulse beams ${ }^{1,3}$ shown in Fig.1-7. Since the modal equations are not valid in the intersection region of the two beams (amplitude and phase variation in space become comparable), we evaluate only the intensities of the pulses up to the lower boundary of the intersection regions and add the field amplitudes at that intersecting point to determine the electron density. As shown in Fig. 1-8, when the two pulses intersect at about $50.2 \mathrm{~km}$, the electron density increases quickly to about $6.6 \times 10^{8} \mathrm{~cm}^{-3}$. This density level is considered to be high enough for practical applications. 




Fig.1-6 Electron density $\left(\mathrm{cm}^{-3}\right)$ distribution generated by focused beams








Fig.1-7 Two crossed microwave beams scheme. 


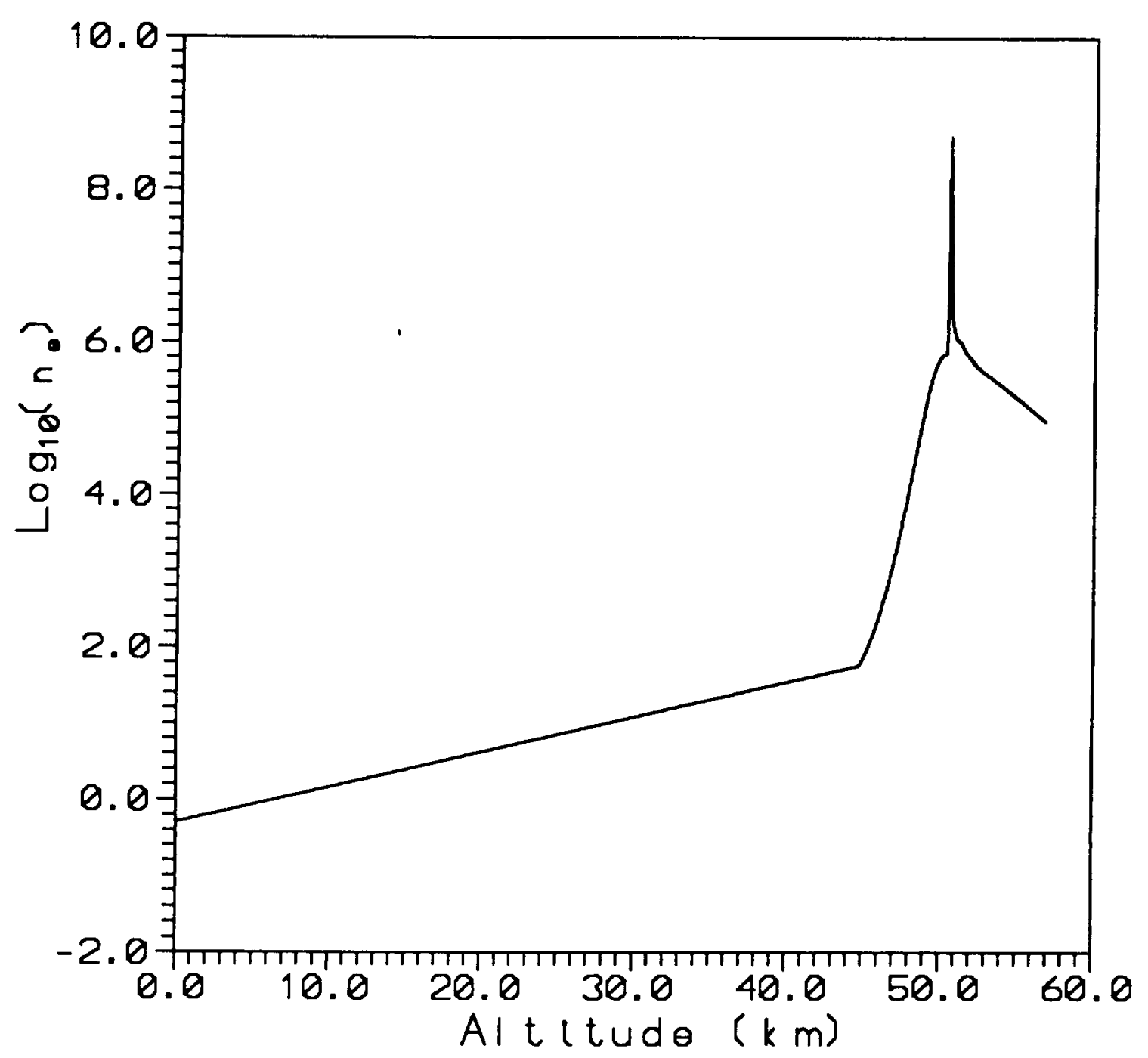

Fig.1-8 Electron density $\left(\mathrm{cm}^{-3}\right)$ distribution generated by two intersecting pulses at $50 \mathrm{~km}$ height. Each pulse has the same parameters as those of Fig.1-5. 


\section{1-4. Summary and Conclusion}

We have investigated numerically the extent of the electron density at a distant altitude location which can be generated by a high power ground transmitted microwave pulse. This is done by varying the power, width, shape and carrier frequency of the pulse. The results show that once the breakdown threshold field is exceeded in the region below the desirable altitude location, electron density starts to build up in that region through cascading breakdown. The generated plasma attenuates the pulse energy (tail erosion) and, thus, deteriorates the energy transmission to the destined altitude. The electron density saturates at a level limited by the pulse width and the tail erosion process. As the pulse continues to travel upward, though the breakdown threshold field of the background air decreases, but the pulse energy (width) is reduced more severely by the tail erosion process. Thus, the electron density grows more quickly at the higher altitude, but saturates at a lower level. Consequently, the maximum electron density produced by a single pulse at $50 \mathrm{~km}$ altitude, for instance, is found by the present study to be limited to a value below $10^{5} \mathrm{~cm}^{-3}$.

A repetitive pulse approach is then considsred. The result indicates that using a sequence of pulses can only slightly increase the electron density at the desirable location. Again, this limitation is caused by the tail erosion process. This leads to the conclusion that air breakdown in the region below the desirable altitude must be avoided so that sufficient pulse energy can be delivered to the desirable region for plasma generation. This can be done by the following two approaches. One uses a focused beam so that only near the given altitude does the field amplitude exceed the local breakdown threshold field. However, this approach requires that the beam focusing rate be much 
greater than the decreasing rate of the breakdown threshold field with the altitude. Thus, it requires a very large antenna array that may not be practical. Moreover, to keep the area of the ionization layer at a practical size one has to limit the focusing effect to, for instance, a power amplification factor of 10 at the desirable altitude. In this case, the ionization is found to be enhanced only by less than an order of magnitude from the unfocused beam case.

The other scheme uses two beams (pulses) intersecting at a given altitude $^{1,3}$. Each beam has a field amplitude below the breakdown threshold of the gas at that altitude. However, the fields in the intersecting region can interfere constructively and exceed the breakdown threshold. This approach is most effective when the two beams are coherent and have the same polarization. In this case, the intersecting wave fields form a standing wave pattern in the direction perpendicular to the bisecting line of the angle $\phi$ between the two beams. Thus parallel plasma layers (instead of a single layer) with a separation $d=\lambda_{0} / 2 \sin (\phi / 2)$ can be generated, where $\lambda_{0}$ is the wavelength of the two beams ${ }^{3}$. Using this approach, it is shown that the electron density at the desired altitude (e.g. $50 \mathrm{~km}$ ) can be increased to about $6.6 \times 10^{8} \mathrm{~cm}^{-3}$, which is considered to be high enough for AIM applications. Since a reflector produced by this approach is a set of parallel layers, it can effectively scatter signals even having their frequencies higher than the plasma cutoff frequency as long as the Bragg scattering condition can be approximately matched ${ }^{3}$. Another advantage of this approach is that the location and the tilted angle of the reflector can be controlled easily by adjusting the incident angles of the two pulse beams. 


\section{References:}

1. Gurevich, A.V., "Ionization of the lower ionosphere under the effect of strong radio pulses," Geomagn. Aeronom., 19, 428-432, 1979.

2. Gurevich, A.V., "An ionized layer in a gas (in the atmosphere)," Sov. Phys. Usp., 23, 862, 1980.

3. Kuo, S.P. and Y.S. Zhang, "Bragg scattering of electromagnetic wave by microwave produced plasma layers," Phys. Fluids B, 2(3), 667-673, 1990.

4. Headrick, J.M. and M.I. Skolnik, "Over-the-Horizon Radar in the HF Band," Proceedings of IEEE vol.62 No.6, 664-673, 1974.

5. Kuo, S.P., Y.S. Zhang, M.C. Lee, P. Kossey and R.J. Barker, "Laboratory chamber experiments exploring the potential use of artificially ionized layer of gas as a Bragg reflector for over-the-horizon signals," Radio Sci., 27(6), 851-865, 1992.

6. Bollen, W.M., C.L. Yee, A.W. Ali, M.J. Nagurney, and M.E. Read, "Highpower microwave energy coupling to nitrogen during breakdown," J. Appl. Phys. 54, 101-106, 1983.

7. Goldstein, B. and C. Longmire, "Microwave absorption and plasma heating due to microwave breakdown in the atmosphere," J. Radiation Effects Res. and Eng., 3, 1626-1628, 1984.

8. Yee, C.L., A.W. Ali, and W.M. Bollen, "Microwave coupling in a nitrogen-breakdown plasma," J. Appl. Phys.,54, 1278-1283, 1983.

9. Yee, J.H., R.A. Alvarez, D.J. Mayhall, N. K. Madsen, and H.S. Cabayan, "Dynamic characteristics of intense short microwave propagation in an atmosphere," J. Radiation Effects Res. and Eng., 3, 152-160, 1984. 
10. Yee, J.H., R.A. Alvarez, D.J. Mayhall, D.P. Byrne, and J. DeGroot, "Theory of intense electromagnetic pulse propagation through the atmosphere," Phys. Fluids, 29, 1238-1244, 1986.

11. Kuo, S.P., Y.S. Zhang, and P. Kossey, "Propagation of high power microwave pulses in air breakdown environment," J. Appl. Phys., 67(6), 2762-2766, 1990a.

12. Kuo, S.P., and Y.S. Żhang, "A theoretical model for intense microwave pulse propagation in an air breakdown environment," Phys. Fluids B, 3(10), 2906-2912, 1991.

13. Hindmarsh, A.C., "LSODE and LSODI, two initial value ordinary differential equations solvers," ACM-SIGNUM newsletter, Vol.15 No.4, 10-11, 1980.

14. Lupan, Yu.A., "Refined theory for an RF discharge in air," Sov. Phys. Tech. Phys., 21(11), 1367-1370, 1976.

15. Davidson, R.C., "Methods in Nonlinear Plasma Theory," Academic Press, 1972. 


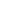

